\title{
Chromobacterium haemolyticum Pneumonia Associated with Near-Drowning and River Water, Japan
}

Hajime Kanamori, Tetsuji Aoyagi, Makoto Kuroda, Tsuyoshi Sekizuka, Makoto Katsumi, Kenichiro Ishikawa, Tatsuhiko Hosaka, Hiroaki Baba, Kengo Oshima, Koichi Tokuda, Masatsugu Hasegawa, Yu Kawazoe, Shigeki Kushimoto, Mitsuo Kaku

We report a severe case of Chromobacterium haemoIyticum pneumonia associated with near-drowning and detail the investigation of the pathogen and river water. Our genomic and environmental investigation demonstrated that river water in a temperate region can be a source of $C$. haemolyticum causing human infections.

Chromobacterium is a genus of gram-negative, facultative anaerobic bacteria; application of $16 \mathrm{~S}$ rRNA gene sequencing into bacterial taxonomy is expanding its species (1-5). Most Chromobacterium infections in humans have been caused by $C$. violaceum (6). Recently, exceptionally rare cases of C. haemolyticum infections have been described (2,4,7-9), but environmental sources of this pathogen have not been well investigated. We describe a case of Chromobacterium-associated pneumonia due to near-drowning and environmental investigation of a river site of the near-drowning. We used whole-genome sequencing (WGS) to identify the Chromobacterium species causing pneumonia associated with near-drowning and investigate molecular features, including antimicrobial resistance, virulence, and genetic relatedness of clinical and environmental isolates of C. haemolyticum.

\section{The Study}

This study was approved by the institutional review board of Tohoku University Graduate School of

\footnotetext{
Authors affiliations: Tohoku University Graduate School of Medicine, Sendai, Japan (H. Kanamori, T. Aoyagi, M. Katsumi, K. Ishikawa, T. Hosaka, H. Baba, K. Oshima, K. Tokuda, M. Hasegawa, Y. Kawazoe, S. Kushimoto, M. Kaku); National Institute of Infectious Diseases, Tokyo, Japan (M. Kuroda, T. Sekizuka)
}

DOI: https://doi.org/10.3201/eid2609.190670
Medicine (IRB no. 2018-1-716). In June 2018, a man in his 70s was transported to our emergency center. He had altered consciousness and hypothermia at admission. He had fallen down a bank and into a river in the Tohoku region of Japan while intoxicated from alcohol and was found immersed in the river. He had respiratory failure and required intubation and mechanical ventilation. He had multiple fractures and a cervical cord injury. He had a history of hypertension, diabetes, and benign prostatic hyperplasia but was not immunodeficient. We diagnosed severe aspiration pneumonia and sepsis and treated the patient empirically with intravenous meropenem plus levofloxacin. We detected a nonpigmented, $\beta$-hemolytic gram-negative bacillus from both sputum and blood cultures. C. violaceum was identified by a matrix-assisted laser desorption/ ionization time-of-flight mass spectrometry (VITEK MS; bioMérieux, https://www.biomerieux.com) with a confidence value of $99.9 \%$. We changed the antimicrobial drug regimen to intravenous ceftazidime plus levofloxacin based on antimicrobial susceptibility testing pattern (Appendix 1 Table, https:/ / wwwnc.cdc.gov/EID/article/26/9/190670-App1.pdf). After 3 weeks of intravenous therapy and critical care, the patient showed clinical improvement and had negative blood and sputum cultures. He was transferred to a community hospital for further rehabilitation and completed an additional 2 months of oral levofloxacin.

In mid-August, we conducted an environmental investigation of the river water in the area where the patient was found. We collected $500 \mathrm{~mL}$ samples of river water, 2 samples at the site where the patient was found and 1 sample $4 \mathrm{~km}$ upstream, where he likely fell into the river. We filtered samples through a 
polyethersulfone filter membrane with a pore size of $0.22 \mu \mathrm{m}$. We placed the membrane filters on sheep blood agar plates and incubated for 24 hours at $35^{\circ} \mathrm{C}$. We recovered a nonpigmented, $\beta$-hemolytic colony similar to clinical isolates from each of the cultures, which we identified as C. violaceum. We performed antimicrobial susceptibility testing by using a MicroScan WalkAway 96 plus (Beckman Coulter, https:/ / www.beckmancoulter.com; Appendix 1) and assessed antimicrobial susceptibility patterns of Chromobacterium isolates (Appendix 1 Table).

We performed WGS on the 3 environmental and 2 clinical isolates (Appendix 1). For comparative genomic analysis, we used additional 16 genome sequences of Chromobacterium spp. from wastewater treatment plants in Tokyo and 52 publicly available genome sequences of Chromobacterium spp. from the NCBI Assembly database (https://www.ncbi. nlm.nih.gov/assembly) (Figure 1; Appendix 1; Appendix 2 Table 1, https://wwwnc.cdc.gov/EID/ article/26/9/19-0670-App2.xlsx). We identified 19 strains of C. haemolyticum with 252,974 single-nucleotide variants by core-genome phylogenetic analysis (Figure 1; Appendix 2 Table 2). Metagenomic analysis of a river water sample collected from the site of the patient's near-drowning revealed that the relative abundance of Chromobacterium is $0.07 \%$ (Figure 2). We deposited the complete genomic sequence of C. haemolyticum CH06-BL in GenBank (accession no. AP019312).

\section{Conclusions}

This severe case of drowning-associated pneumonia and bacteremia due to C. haemolyticum was successfully treated with appropriate antimicrobial therapy. Previously, 5 clinical cases of C. haemolyticum infections had been reported, including sputum colonization, necrotizing fasciitis with bacteremia, proctocolitis, pneumonia, and pediatric bacteremia (2,4,7-9). All patients, including the patient we report, survived after antimicrobial treatment. Intravenous antimicrobial therapy, such as meropenem or fluoroquinolone, is recommended for C. haemolyticum infections $(7,9)$. The role of prolonged therapy for $C$. haemolyticum infections remains unclear, but in C. violaceum infections, an oral agent such as trimethoprim-sulfamethoxazole, tetracycline, or fluoroquinolone for 2-3 months can be used to prevent relapse (6).

As seen in the case we report, identification of Chromobacterium species is challenging. C. violaceum can produce a violet pigment (violacein) in most strains, and nonpigmented strains rarely have been

\begin{tabular}{|c|c|c|c|c|c|c|}
\hline \multirow{2}{*}{ SNV tree } & Strain name & Year & Place & Source & Material & BioSample \\
\hline & $\mathrm{H} 4137$ & 2006 & United States & Human & Finger & SAMN06294330 \\
\hline & IR17 & 2012 & Portugal & Environment & Water & SAMN04407340 \\
\hline & - DSM 19808 & 2007 & United States & Human & Sputum & SAMN02745482 \\
\hline & - H5244 & 2009 & United States & Human & Trachea & SAMN06294331 \\
\hline & H3973 & 2006 & United States & Human & Blood & SAMN06294329 \\
\hline \multirow{3}{*}{0.2} & NRRL B-11053 & N/A & N/A & $N / A$ & N/A & SAMN08868474 \\
\hline & C-61 & N/A & N/A & N/A & N/A & SAMEA2272075 \\
\hline & CH06-BL & 2018 & Japan & Human & Serum & SAMD00149624 \\
\hline \multirow{6}{*}{$\begin{array}{r}\text { Bootstrap } \\
100 \%\end{array}$} & CH06-SPT & 2018 & Japan & Human & Sputum & SAMD00149623 \\
\hline & $\mathrm{T} 124$ & 2013 & Japan & Human & Blood & SAMN02870191 \\
\hline & CH08-RW3 & 2018 & Japan & Environment & River water & SAMD00149627 \\
\hline & CH08-RW1 & 2018 & Japan & Environment & River water & SAMD00149625 \\
\hline & JP2-74 & 2017 & China & Rice & Rhizosphere & SAMN09925372 \\
\hline & GAS-1-3-11 & 2017 & Japan & Environment & Sewage effluent & SAMD00149530 \\
\hline \multirow{5}{*}{$\begin{array}{l}\text { Reference: } \mathrm{CHO6-BL}(5,307,994 \mathrm{bp}) \\
\text { Core genome region: } 64.15 \% \\
\text { Total SNVs: } 252,974 \text { sites }\end{array}$} & CH08-RW2 & 2018 & Japan & Environment & River water & SAMD00149626 \\
\hline & GAR-3-9-4 & 2018 & Japan & Environment & Sewage effluent & SAMD00149531 \\
\hline & GSH-3-8-14 & 2018 & Japan & Environment & Sewage effluent & SAMD00149532 \\
\hline & GAR-1-9-10 & 2017 & Japan & Environment & Sewage effluent & SAMD00149528 \\
\hline & GAR-1-9-7 & 2017 & Japan & Environment & Sewage effluent & SAMD00149529 \\
\hline
\end{tabular}

Figure 1. Core genome single-nucleotide variations in a phylogenetic analysis of 19 strains of Chromobacterium haemolyticum in a case of pneumonia associated with near-drowning in river water, Japan. In total, 252,974 SNV sites were detected in core genome region among 19 strains. The phylogenetic analysis with SNV data was constructed by maximum likelihood method. Two clinical isolates (CH06-BL and CH06-SPT) and 3 environmental isolates (CH08-RW1, CH08-RW2, and CH08-RW3) of C. haemolyticum in this study were discordant $(27,867-29,491$ SNVs). Scale bar indicates nucleotide substitutions per site. SNV, single nucleotide variant. 


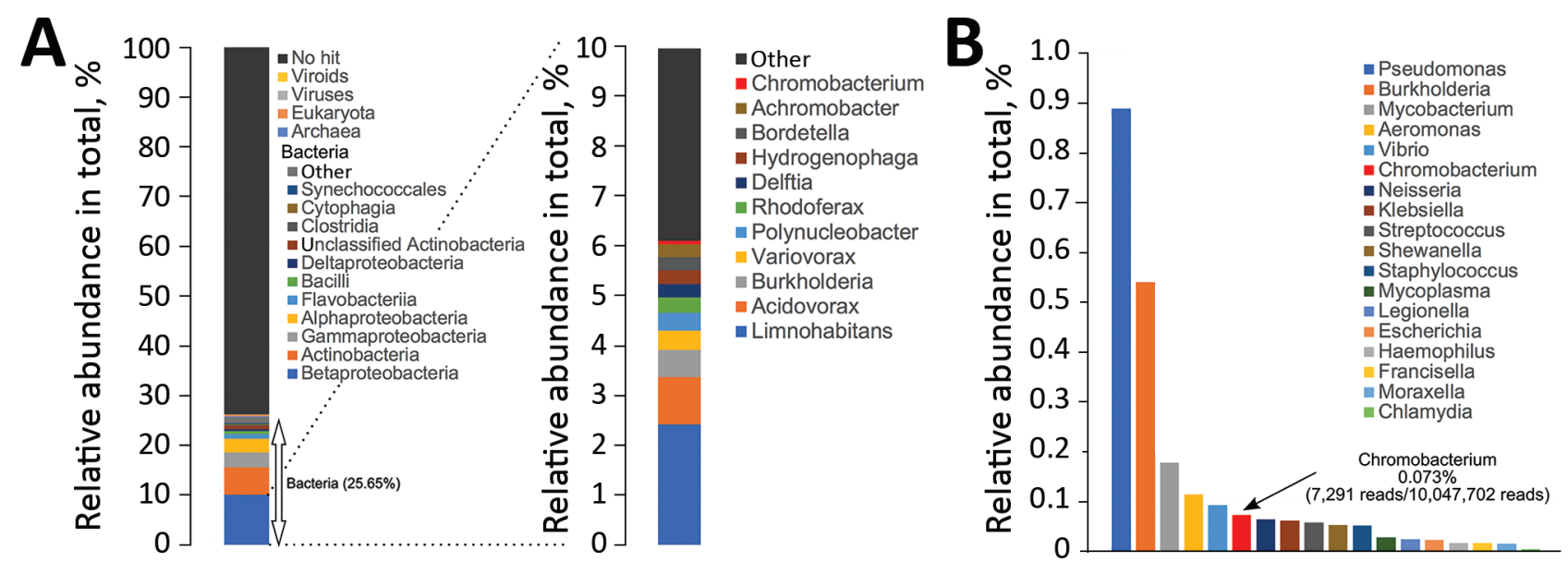

Figure 2. Metagenomic analysis of river water sample collected from the site of near-drowning of a patient with Chromobacterium haemolyticum pneumonia, Japan. A) Relative abundance of superkingdom, class of bacteria, and genus of betaproteobacteria in river water sample. The relative abundance of bacteria is $25.65 \%$; the 10 most observed class and genus are summarized in cumulative bar charts. B) Comparison of relative abundance of bacteria causing pneumonia associated with drowning in genus level in the river water sample. The relative abundance of Chromobacterium, a Betaproteobacteria, is $0.073 \%$.

described (10). C. haemolyticum does not produce violacein and is characterized by strong hemolytic activity on sheep blood agar $(2,4)$. Only C. violaceum is currently available in the genus Chromobacterium on the mass spectrometry database of species identification. Differentiation between C. haemolyticum and C. violaceum is crucial because $C$. haemolyticum has greater resistance to antimicrobial drugs, such as $\beta$-lactams $(2,7)$. Although $C$. aquaticum is a nonpigmented, $\beta$-hemolytic strain phenotypically similar to $C$. haemolyticum, 16S rRNA sequencing might not determine either $C$. haemolyticum or $C$. aquaticum because of artificial separation of both species (4). Thus, WGS is a useful tool for accurate identification of Chromobacterium species to avoid misidentification of $C$. haemolyticum (1-5).

C. haemolyticum CH06-BL and other clinical and environmental isolates in this study possessed $b l a_{\mathrm{CRH}-1}$ in the chromosome (Appendix 2 Table 1), but we did not identify mobile elements in the surrounding area. In a previous study, a class A $\beta$-lactamase, CRH-1 from $C$. haemolyticum was closely related to Klebsiella pneumoniae carbapenemase 2 (11). As seen in acquired resistance among other gram-negative bacilli, aquatic environments can be a reservoir $(11,12)$.

The etiology of infections caused by Chromobacterium has not been fully elucidated. Of note, Chromobacterium accounted for only a small portion of the bacteria found in our metagenomics analysis of the river water, but this organism was isolated from the patient and was involved in human infection, despite presence of other potential pathogens in the river, such as Pseudomonas, Aeromonas, Legionella, that can cause pneumonia associated with drowning (Figure 2) (13). Our study isolates also had type III secretion system (T3SS) encoded by Chromobacterium pathogenicity island 1 and $1 \mathrm{a}$ (Cpi-1/-1a) (Appendix 1 Figure $2)$, which is known as a major virulence factor in $C$. violaceum (14). These results highlight the need for further research on antimicrobial resistance and virulence in Chromobacterium spp.

C. violaceum is widely distributed in natural aquatic environments and can be observed in water and soil sources, especially in tropical and subtropical areas (6). C. haemolyticum strains with genetic heterogeneity have been detected from lake water in a tropical region (15), but the bacterium's habitat in temperate regions remains unknown. Our comparative genomic analysis revealed that clinical and environmental isolates of $C$. haemolyticum were discordant (27,867-29,491 single-nucleotide variants), although there was no standard definition for its clonality.

Only 2 reports of cases with C. haemolyticum infections in temperate regions of Japan have been published $(7,9)$. One study reported necrotizing fasciitis associated with exposure to river water after injury. The other described pneumonia caused by accidental aspiration of runoff water after a fall in a ditch and identification of the pathogen in the water and discordant results with clinical isolates by pulsed-field gel electrophoresis. However, detailed environmental investigations of the rivers as a source of the pathogen were not conducted in either article.

In summary, our genomic and environmental study demonstrates that $C$. haemolyticum in a local river, a natural habitat of this pathogen in Japan, caused 
this human infection. Clinicians should remain aware that river water in temperate regions can be a source of C. haemolyticum infection.

\section{Acknowledgments}

We thank David J. Weber for his careful review of the manuscript.

This work was supported in part by a grant for the Research on Emerging and Re-emerging Infectious Diseases and Immunization (grant no. H30

Shinkogyosei-Ippan-002) from the Ministry of Health, Labour and Welfare, Japan, and a grant from the Research Program on Emerging and Re-emerging Infectious Diseases from the Japan Agency for Medical Research and Development (grant nos. JP18fk0108048 and JP18fk0108019).

\section{About the Author}

Dr. Kanamori is an infectious disease physician at Tohoku University Hospital, Sendai, Japan. His primary research interests are antimicrobial resistance, environmental hygiene, and healthcare epidemiology.

\section{References}

1. Blackburn MB, Farrar RR Jr, Sparks ME, Kuhar D, Mitchell A, Gundersen-Rindal DE. Chromobacterium sphagni sp. nov., an insecticidal bacterium isolated from Sphagnum bogs. Int J Syst Evol Microbiol. 2017;67:3417-22. https:/ / doi.org/10.1099/ijsem.0.002127

2. Han XY, Han FS, Segal J. Chromobacterium haemolyticum sp. nov., a strongly haemolytic species. Int J Syst Evol Microbiol. 2008;58:1398-403. https:// doi.org/10.1099/ijs.0.64681-0

3. Bajaj A, Kumar A, Yadav S, Kaur G, Bala M, Singh NK, et al. Isolation and characterization of a novel Gram-negative bacterium Chromobacterium alkanivorans sp. nov., strain IITR-71T degrading halogenated alkanes. Int J Syst Evol Microbiol. 2016;66:5228-35. https://doi.org/10.1099/ ijsem.0.001500

4. Harmon N, Mortensen JE, Robinette E, Powell EA. Pediatric bacteremia caused by Chromobacterium haemolyticum / Chromobacterium aquaticum. Diagn Microbiol Infect Dis. 2016;86:108-11. https:// doi.org/10.1016/j.diagmicrobio. 2016.05.021
5. Zhou S, Guo X, Wang H, Kong D, Wang Y, Zhu J, et al. Chromobacterium rhizoryzae sp. nov., isolated from rice roots. Int J Syst Evol Microbiol. 2016;66:3890-6. https:/ / doi.org/10.1099/ijsem.0.001284

6. Yang CH, Li YH. Chromobacterium violaceum infection: a clinical review of an important but neglected infection. J Chin Med Assoc. 2011;74:435-41. https:/ / doi.org/ 10.1016/j.jcma.2011.08.013

7. Okada M, Inokuchi R, Shinohara K, Matsumoto A, Ono Y, Narita M, et al. Chromobacterium haemolyticum-induced bacteremia in a healthy young man. BMC Infect Dis. 2013;13:406. https://doi.org/10.1186/1471-2334-13-406

8. Tanpowpong P, Charoenmuang R, Apiwattanakul N. First pediatric case of Chromobacterium haemolyticum causing proctocolitis. Pediatr Int. 2014;56:615-7. https://doi.org/10.1111/ped.12301

9. Takenaka R, Nureki S, Ueno T, Shigemitsu O, Miyazaki E, Kadota J, et al. Chromobacterium haemolyticum pneumonia possibly due to the aspiration of runoff water. Jpn J Infect Dis. 2015;68:526-9. https:/ / doi.org/10.7883/ yoken.JJID.2014.285

10. Yang CH. Nonpigmented Chromobacterium violaceum bacteremic cellulitis after fish bite. J Microbiol Immunol Infect. 2011;44:401-5. https:// doi.org/10.1016/j.jmii.2010.04.004

11. Gudeta DD, Bortolaia V, Jayol A, Poirel L, Nordmann P, Guardabassi L. Chromobacterium spp. harbour Ambler class A $\beta$-lactamases showing high identity with KPC. J Antimicrob Chemother. 2016;71:1493-6. https://doi.org/10.1093/jac/ dkw020

12. Tacão M, Correia A, Henriques IS. Low prevalence of carbapenem-resistant bacteria in river water: resistance is mostly related to intrinsic mechanisms. Microb Drug Resist. 2015;21:497-506. https:// doi.org/10.1089/mdr.2015.0072

13. Ender PT, Dolan MJ. Pneumonia associated with near-drowning. Clin Infect Dis. 1997;25:896-907. https://doi.org/10.1086/515532

14. Miki T, Okada N. Draft genome sequence of Chromobacterium haemolyticum causing human bacteremia infection in Japan. Genome Announc. 2014;2:e01047-14. https://doi.org/10.1128/ genomeA.01047-14

15. Lima-Bittencourt CI, Costa PS, Barbosa FA, Chartone-Souza E, Nascimento AM. Characterization of a Chromobacterium haemolyticum population from a natural tropical lake. Lett Appl Microbiol. 2011;52:642-50. https:/ / doi.org/10.1111/ j.1472-765X.2011.03052.x

Address for correspondence: Hajime Kanamori; Department of Infectious Diseases, Internal Medicine, Tohoku University Graduate School of Medicine, 1-1 Seiryo-machi, Aoba-ku, Sendai 980-8574, Japan; email: kanamori@med.tohoku.ac.jp 